“(C) 2019 IEEE. Personal use of this material is permitted. Permission from IEEE must be obtained for all other uses, in any current or future media, including reprinting/republishing this material for advertising or promotional purposes, creating new collective works, for resale or redistribution to servers or lists, or reuse of any copyrighted component of this work in other works." 


\title{
Electricity Price Forecast using Meteorology data: a study in Australian Energy Market
}

\author{
Ming Zhao ${ }^{1}$, Yangyang $\mathrm{Shu}^{1}$, Shaowu $\mathrm{Liu}^{1}$, Guandong $\mathrm{Xu}^{1 *}$ \\ ${ }^{1}$ Advanced Analytics Institute, University of Technology Sydney. \\ ming.zhao@uts.edu.au, yangyang.shu@student.uts.edu.au, \{shaowu.liu, guandong.xu\}@uts.edu.au
}

\begin{abstract}
Electricity price as a fundamental cost for each family which is an essential segment in the electricity market. The adjustment of electricity price can present the change in electricity supply and demand relationship. For the electricity supply companies, an appropriate defined electricity price can eventually determine the level of profit. On the other hand, an accurate prediction can help to seize opportunities in the electricity market. In this paper, we aim to predict the electricity price with more confident accuracy by leveraging data mining techniques. Our experiment on 12 months of electricity prices as well as climate data in the New South Wales has achieved a promising prediction result.
\end{abstract}

Index Terms-Electricity Price Forecast, Forecasting, Australian Energy Market

\section{INTRODUCTION}

Electricity now is being widely used in everywhere, electricity price is a key factor in the electricity market, which ensures steady operation of the entire market. For the electricity supply companies, the forecast of electricity price has become a critical research area. However, traditional way of defining electricity price usually requires tedious researches, which also needs various analysts with additional costs. This outdated process can hardly make contributions to formulate an accurate bidding plan by the prediction of electricity price, and eventually lost the market shares and great profits [2]. Meanwhile, by using a confident electricity price forecast, the company can effectively adjust the electricity supply to achieve a balance between supply and demand, and eventually can obtain the greatest benefits; for the customers, they could easily get a purchase costs of electricity consumption through predicting electricity price. One of the major issues within this forecast is that electricity price has a certain periodicity and volatility, plus the effectiveness of quantifiable factors, such as historical electricity price and climate change, etc. All these issues make the electricity price forecast become difficult.

Applying machine learning techniques can make it possible to predict the electricity price more effectively [3]. Specifically, the prediction of electricity price can be considered as a regression problem in machine learning.

Before discussing the technical details, we would like to emphases the importance of electricity price prediction. The energy market in Australia is an open market with large number of companies selling energy produced from different sources, including coal, gas, wind, and solar power. Consequently, the market behaves like a "stock market" and companies need to "guess" the price to decide their production strategy. Be able to know the electricity price in advance will help all players in the market to dynamically change their production strategy to avoid the wasting of power as well as overloaded power grid, resulting a healthier yet dynamic energy market.

\section{Preliminaries}

In Australia, electricity price is made up of four major components, including:

Wholesale costs cover the cost which electricity being generated, e.g., coal, solar power, etc.

Network charges are used to pay for the reliable delivery of energy via power grids to residential properties.

Retail costs/margin are the cost of paying for meter reading, bill management, and maintenance services.

Environmental costs include Australian state and territory environmental programs, e.g., a program aims to increasing renewable electricity generation.

In recent years, the policies to address environmental issues have attributed a lot to the electricity price increases. Another big impact to the prices is the investment of network component, due to previously the under-investment to maintain the network operations or the capacity increment. While the smaller electricity price increases are largely due to the changes to the regulation of electricity networks and competition in electricity markets.

\section{MODEL}

Electricity price in Australia is dynamic and changes everyday. The price is affected by various factors including both customer-related and environment-related. In this work, we propose to use machine learning regression models to model the meteorology information for predicting daily electricity prices. To be specific, the following five models are employed.

Linear Regression attempts to model the relationship between two variables by fitting a linear equation to observed data.

Ridge Regression is a regression model particularly useful for modelling multiple regression data that suffer from multicollinearity.

Lasso Regression is a type of linear regression that uses shrinkage, where data values are shrunk towards a central point. Lasso Regression encourages simple models. 


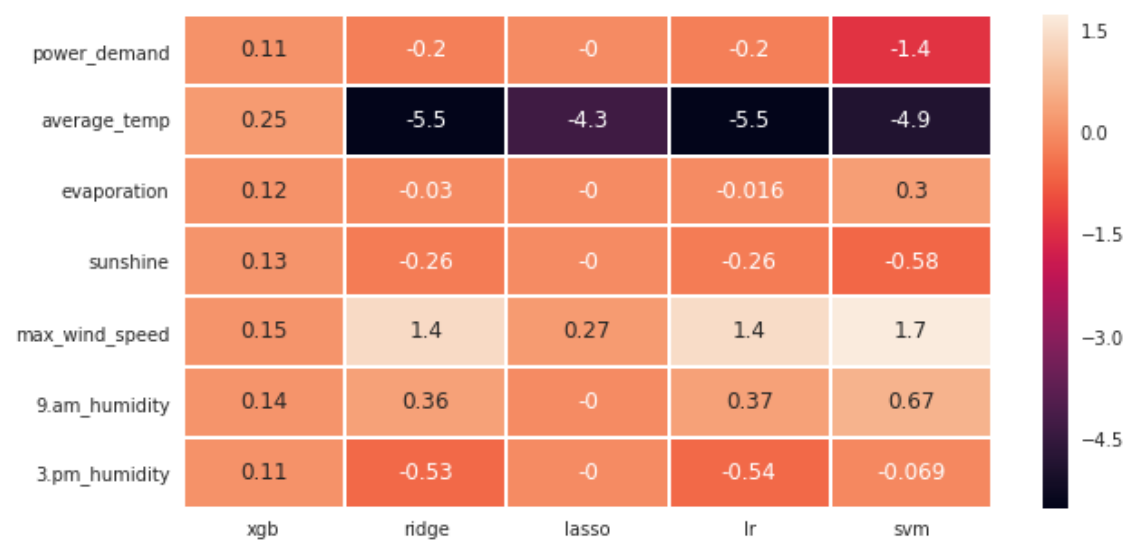

Fig. 1. Feature Importance

SVM Regression uses the same principles as the Support Vector Machines with minor modifications.

XGBoost Regression [1] builds an ensemble of weak prediction models via gradient boosting procedure.

Root Mean Square Error (RMSE) will be used as the major evaluation metric to compare the prediction result from above five models, which measures of how far from the regression line data points are.

$$
R M S E=\sqrt{\frac{1}{n} \sum_{i=1}^{n}\left(f_{i}-d_{i}\right)^{2}}
$$

where $f_{i}$ and $d_{i}$ are the predicted and actual value.

\section{EXPERIMENT}

The experiment was conducted on a 365 days dataset with 7 features between 2018 and 2019 in New South Wales, Australia. The data collection includes electricity demand, average temperature, evaporation, sunshine, maximum wind speed at 9AM, humidity at 3PM, plus the electricity price on each day.

Evaluation result on 10-fold cross validation is given in Table I.

\begin{tabular}{c|c|c|c|c|c}
\hline Metric & xgb & ridge & lasso & lr & linear svm \\
\hline RMSE & 11.16 & 11.42 & 10.98 & 11.42 & 12.61 \\
\hline
\end{tabular}

TABLE I: Regression result on RMSE

To explore the impact of each feature on the electricity price, we also extracted feature importance or coefficients from the trained models, as shown in Table II and Figure 1.

We observe that among all features, average temperature shows the strongest impact on electricity price, followed by maximum wind at 9AM.

\begin{tabular}{l|c|c|c|c|c}
\hline Feature & $\boldsymbol{x g} \boldsymbol{b}$ & ridge & lasso & lr & linear svm \\
\hline power_demand & 0.109 & -0.2 & 0 & -0.202 & -1.425 \\
\hline average_temp & 0.245 & -5.5 & -4.318 & -5.526 & -4.782 \\
\hline evaporation & 0.122 & -0.03 & 0 & -0.016 & 0.164 \\
\hline sunshine & 0.133 & -0.261 & 0 & -0.258 & -0.718 \\
\hline max_wind & 0.149 & 1.427 & 0.265 & 1.431 & 1.751 \\
\hline 9am_humidity & 0.137 & 0.358 & 0 & 0.373 & 0.658 \\
\hline 3pm_humidity & 0.105 & -0.535 & 0 & -0.545 & -0.154 \\
\hline
\end{tabular}

TABLE II: Feature importance/coefficients.

\section{CONCLUSIONS}

In this paper we proposed to use several regression models to predict electricity price based on specified input features. By applying these methods to a 365 days daily electricity price dataset, Lasso regression and XGBoost regression achieved the lowest RMSE so far with a better prediction result than other models. Meanwhile, in the given seven input features, average temperature and max wind speed are the most important features which has strong impact to the electricity price. These findings will benefit Australian electricity supply companies to better define electricity price and strategy.

\section{ACKNOWLEDGMENT}

The authors would like to thank Australian Energy Market Commission (AEMC) and Bureau of Meteorology (BOM) for providing this invaluable dataset.

\section{REFERENCES}

[1] Tianqi Chen and Carlos Guestrin. Xgboost: A scalable tree boosting system. In Proceedings of the 22nd acm sigkdd international conference on knowledge discovery and data mining, pages 785-794. ACM, 2016.

[2] Ping-Huan Kuo and Chiou-Jye Huang. An electricity price forecasting model by hybrid structured deep neural networks. Sustainability, 10(4):1280, 2018.

[3] Can Wan, Zhao Xu, Yelei Wang, Zhao Yang Dong, and Kit Po Wong. A hybrid approach for probabilistic forecasting of electricity price. IEEE Transactions on Smart Grid, 5(1):463-470, 2013. 